\title{
Practical considerations in planning a cath-lab
}

\author{
D H Schulenburg, $M B C h B$ \\ Department of Radiology, Universitas Hospital, Bloemfontein and University of \\ the Free State
}

Vascular laboratories play an increasing part in treating patients via intravascular procedures. The diagnostic purpose of vascular laboratories is being somewhat diminished by excellent diagnostic imaging machines like the 64-slice CT scanner and three Tesla MRI scanners. Thus, the emphasis falls on treating patients using intravascular procedures rather than performing diagnostic procedures. When planning a vascular laboratory it should be kept in mind that the main purpose of such a laboratory is to treat patients using various interventional skills.

Planning a vascular laboratory depends on various factors like the geographic environment of the radiology practice, the need for interventional procedures, types of angiography procedures, the number of patients, building size, and lastly the budget available for equipment and running costs. Usually the suppliers of the angio-equipment will play an integral part in planning and developing a catheterisation laboratory. Some of the practical planning aspects are discussed in this article.

\section{The vascular laboratory and surrounding rooms}

Apart from fulfilling the requirements of the International Commission on Radiological Protection (ICRP) on X-ray protection, the vascular laboratory should be large enough to accommodate the angio-system. If enough space is available, the laboratory can be developed in such a way that a dual-plane system can be fitted. This will enable conversion of the laboratory into a surgical theatre where vascular procedures can be performed after the diagnostic work has been completed, especially in critical cases and where theatre space is a problem.

The vascular laboratory should be planned in such a way that patient flow is optimum. Incoming and outgoing patients should not interfere with each other. A waiting room for incoming patients, especially outpatients, is essential. This room can also be used to explain procedures to patients and their families. Changing rooms and toilet facilities for patients should be close by.

A recovery room equipped with oxygen connections, ECG machines and an anaesthetic machine must be in close proximity of the vascular lab, especially if patients receive anaesthesia during procedures.

Each vascular lab must have a computer room with separate airconditioning. This room hosts the C-arm power supply cabinets, as well as X-ray tube heat converters and any other computer hardware necessary for the vascular lab. The control room should be state of the art with huge glass windows that overlook the vascular laboratory so that effective handling of software occurs during a procedure. A separate entrance into the control room gives access to personnel without the risk of accidental radiation in the vascular laboratory. Enough space must be available for the computer hardware needed for the angio-system.

Storerooms are required for bulky equipment, as well as products used in the vascular laboratory during procedures. One of these storerooms can be converted into a setting-up room where sterile trays are prepared for interventional procedures. A lockable cupboard in the setting-up room can be used for storing anaesthetic drugs.

A separate entrance away from the waiting and recovery room is ideal for disposal of soiled linen and waste material. Cleaning services can also use this entrance for access to clean the theatre after each procedure.

Changing rooms equipped with lockers and shower and scrubbing facilities for the radiologist/cardiologist should be available just outside the vascular laboratory.

\section{Vascular laboratory}

The vascular laboratory should be equipped with excellent lighting. Lights should automatically switch off during fluoroscopy. A small ceiling-mounted light is practical during difficult catheterisation procedures, i.e. when brachial artery entrance is used or when putting up IV canulas in patients with 'difficult' veins, i.e. children and cancer patients. A theatre light should be suspended from the ceiling in such a way that there is no interference with the $\mathrm{C}$-arms but close enough so that the angiographic table can be used as an operating table. Gas fittings should also be available to connect to an anaesthetic machine. Enough power sockets are essential to connect different apparatus in the vascular theatre, i.e. ECG machine, ultrasound, heating equipment for contrast agents, etc. Some of these power sockets should also be connected to an emergency power supply in case of power loss.

\section{Angio-equipment}

There is a choice between a single-plane and dual-plane system. The dual-plane concept is gaining tremendous acceptance throughout the industry. Numerous health-care facilities just do not have the patient volume to justify a separate cardiac lab and a separate vascular-imaging suite. Dual-plane is the perfect solution. Dual-plane incorporates the use of two hyperspeed C-arms that share a common angiographic table. One $\mathrm{C}$-arm can then be equipped with a 9-inch image intensifier that is optimal for cardiac imaging and the second C-arm is equipped with a 16inch image intensifier that is optimal for complete vascular imaging. The $\mathrm{C}$-arms and imaging systems are interfaced with a dual-plane software computer program and the result is complete and total optimisation for each physician specialist. The dual-plane C-arms can both be ceiling-suspended or one ceiling-suspended and the other floor-mounted. Different $\mathrm{C}$-arms are on the market with capabilities of producing 3D images with a recording speed of up to 30 frames per second.

A CCD digital camera with 1 million-pixel images is now available for digital fluorography systems. The CCD camera makes it possible to gather extremely accurate image data at high speed using a 1024x1024 matrix.

C-arms equipped with flat-panel detectors (FPDs) are replacing the image-intensifying screens. The FPDs have many advantages over the image intensifier:

- Uniform brightness across image

- Increased resolution and no distortion

- High dynamic range

- Better technique control 
- One powerful PC to review MR, CT, and vascular 3D images.

Radiation exposure is lower with FPD products. This is very important as any decrease in radiation makes a procedure safer for both screening personnel and patients.

Real-time Smooth Masking (RSM, Shimadzu) is very useful when working with unco-operative patients. This offers DSA images that are never unreadable as a result of motion artifacts. Using RSM, you can acquire DSA images in a pendulum motion, a precession motion, or even a C-arm 'Fast Spin' ( $60^{\circ}$ per sec) acquisition all in real time.

The Magnetic Navigation System (Siemens), is the first magnetic catheter navigation system of its kind in the world. The system integrates an advanced digital fluoroscopic imaging system, with FPD system, to direct and digitally control catheter- and guide wire-based devices along complex paths within the heart and coronary vasculature. The system uses computer-controlled permanent magnets external to the body for orientating the magnetic tip of specially designed medical devices, i.e. catheters. This approach has the potential to provide greater precision and better movement and outcomes for patients.

\section{Additional equipment}

A dedicated ultrasound machine is very useful in a vascular laboratory. It can be very helpful during difficult catheterisations and when one is performing various interventional procedures, i.e. kidney biopsies or liver carcinoma ablations. Additional equipment like a defibrillator, refrigerator, oven and light boxes should be available. The angiogram tables are not suitable for CPR and should be stabilised before commencing CPR. This stabilising device should be placed next to the defibrillator.

A contrast pump is essential in the vascular laboratory. The choice between a ceiling- or a floor-mounted unit depends on the size of the vascular laboratory and the type of equipment in the laboratory. The ceiling-mounted unit is practical but it can interfere with the ceilingsuspended C-arm.

\section{DICOM and PACS}

Angiographic equipment is available with a DICOM network interface, meaning that the examination information leaving the system is already formatted according to the DICOM standards. This has two advantages: (1) data equivalence is assured; and (2) any receiving system that supports this interface can be used for storing and reviewing images and data.
The DICOM system can be linked to the picture archiving and communications system (PACS). PACS digital images are stored in computer memory and can be retrieved in seconds. This reduces the time between exam and therapy, and has potential to impact on the overall length of hospital stay. With digital imaging, doctors can immediately look up a patient's new and past images on a PC, instead of waiting for films to be de-archived from a shelf and delivered. In addition, a doctor can electronically transmit a digital X-ray or other images to a hospital or clinic anywhere in the world. The PACS system supports the ability to diagnose across various modalities and lab systems. The network system between DICOM-compatible machines and a PACS should have a 1-gigabyte cable for efficient transfer of data.

New technology exists which can send health-care information wirelessly to wherever it is needed. The system handles not only text, but also images, audio clips, streaming video, and digitised hand-written notes. This system with DICOM-compliant PACS installations, includes an automatic return receipt, audible alerts for incoming messages, and the ability to $\log$ events.

Changing to a digital system creates a filmless network with the following advantages:

- Elimination of film

- Handling and storage costs reduced

- Increased efficiency and exam throughput

- Instant access to previous studies online

- Optimised diagnostic review with true 1024x1024 images acquisition transfer and storage.

A voice-recognition system is a very practical program. This allows the radiologist to dictate directly onto a computer system. With the help of a radiological information system (RIS)/PACS and the hospital information system interface, this report can be immediately available on the hospital network. This method is especially helpful during after-hour procedures when secretaries are not on duty.

\section{Conclusion}

Development of an angio- or vascular laboratory should be planned according to the need for the specific laboratory. Factors like space, the number of potential patients and the budget available for the project play a very important role. Most of these angiographic systems have many upgrade facilities available, especially software products and the PACS system. The planning of the vascular laboratory should be done by a multidisciplinary panel, keeping these factors in mind. 